\title{
REGULARIZATION OF A UNILATERAL OBSTACLE PROBLEM ON THE BOUNDARY
}

\section{A. ADDOU and J. ZAHI}

Received 28 October 2001

\begin{abstract}
We give a regularization method for a unilateral obstacle problem with obstacle
\end{abstract} on the boundary and second member $f$.

2000 Mathematics Subject Classification: 35J85.

1. Introduction. Let $\Omega$ be a bounded domain of $\mathbb{R}^{n}$ with smooth boundary $\Gamma=\partial \Omega$. We consider the variational inequality problem-called obstacle problem: find

$$
u \in H^{1}(\Omega)
$$

such that

$$
\int_{\Omega} \nabla u \nabla(v-u)+\int_{\Omega} u(v-u)+\int_{\Gamma} v^{-}-u^{-}+\langle f, v-u\rangle \geq 0 \quad \forall v \in H^{1}(\Omega),
$$

where $f \in L^{2}(\Omega)$; it is well known that problems (1.1) and (1.2) admit a unique solution (see [3]).

The aim of this paper is to develop a regularization method for solving a nondifferentiable minimization problem that is equivalent to problems (1.1) and (1.2).

The idea of the regularization method is to approximate the nondifferentiable term by a sequence of differentiable ones depending on $(\varepsilon \geq 0, \varepsilon \rightarrow 0)$.

We give three forms of regularization for which we establish the convergence result and a priori error estimates.

Next, by duality method of conjugate functions (see [1]), we provide a posteriori error estimates desired for the numerical computation. And as an application, we develop a regularization method for solving a sequence of penalised problem.

2. Formulation and regularization method. Let $\Omega$ be a bounded domain of $\mathbb{R}^{n}$ with smooth boundary $\Gamma=\partial \Omega$ and let $f \in L^{2}(\Omega)$.

We denote by $\langle\cdot, \cdot \cdot\rangle$ the duality pairing between $H^{-1}(\Omega)$ and $H^{1}(\Omega)$, and $(\cdot, \cdot)$ the inner product of $L^{2}(\Omega)$. 
Consider the following variational inequality problem:

$$
\begin{aligned}
& \text { Find } u \in H^{1}(\Omega), \\
& a(u, v-u)+\int_{\Gamma} v^{-}-u^{-}+\langle f, v-u\rangle \geq 0 \quad \forall v \in H^{1}(\Omega),
\end{aligned}
$$

where $a(\cdot, \cdot)$ is defined by

$$
a(u, v)=\int_{\Omega} \nabla u \cdot \nabla v+\int_{\Omega} u \cdot v, \quad u, v \in H^{1}(\Omega) .
$$

It is well known that problem (2.1) admit a unique solution (see [3]).

For all $z \in L^{2}(\Omega)$, we denote

$$
z^{+}=\max \{z, 0\}, \quad z^{-}=\max \{0,-z\}
$$

If $v \in H^{1}(\Omega)$, then we have $v^{+}, v^{-} \in H^{1}(\Omega)$ and

$$
a\left(v^{+}, v^{-}\right)=0
$$

DEFINITION 2.1. Let $\varphi$ be the following functional:

$$
\varphi(v)=\int_{\Gamma} v^{-}, \quad v \in H^{1}(\Omega)
$$

The functional $\varphi$, being nondifferentiable on $H^{1}(\Omega)$, is approximated by a sequence of differentiable functionals

$$
\varphi_{\varepsilon}(v)=\int_{\Gamma} \phi_{\varepsilon}(v) d x, \quad(\varepsilon \geq 0, \varepsilon \longrightarrow 0)
$$

The regularized problem is

$$
\begin{aligned}
& \text { Find } u_{\varepsilon} \in H^{1}(\Omega), \\
& a\left(u_{\varepsilon}, v-u_{\varepsilon}\right)+\varphi_{\varepsilon}(v)-\varphi_{\varepsilon}\left(u_{\varepsilon}\right)+\left\langle f, v-u_{\varepsilon}\right\rangle \geq 0 \quad \forall v \in H^{1}(\Omega) .
\end{aligned}
$$

Problems (2.1) and (2.7), respectively, are equivalent to

$$
\begin{aligned}
u \in H^{1}(\Omega): & a(u, v-u)+\int_{\Gamma}\left(v^{-}-u^{-}\right) d x \\
& +\int_{\Omega} f(v-u) d x \geq 0 \quad \forall v \in H^{1}(\Omega), \\
u_{\varepsilon} \in H^{1}(\Omega): & a\left(u_{\varepsilon}, v-u_{\varepsilon}\right)+\varphi_{\varepsilon}(v)-\varphi_{\varepsilon}\left(u_{\varepsilon}\right) \\
+ & \int_{\Omega} f\left(v-u_{\varepsilon}\right) d x \geq 0 \quad \forall v \in H^{1}(\Omega) .
\end{aligned}
$$


There are many methods to construct sequences of differentiable approximations. In this paper, we take the sequence $\phi_{\varepsilon}$ verifying one of the following choices:

$$
\begin{aligned}
& \phi_{\varepsilon}^{1}(t)= \begin{cases}0 & \text { if } t \geq 0, \\
\frac{t^{2}}{2 \varepsilon} & \text { if }-\varepsilon \leq t \leq 0, \\
-t-\frac{\varepsilon}{2} & \text { if } t \leq-\varepsilon,\end{cases} \\
& \phi_{\varepsilon}^{2}(t)= \begin{cases}\frac{\varepsilon}{2} & \text { if } t \geq 0, \\
\frac{1}{2}\left(\frac{t^{2}}{\varepsilon}+\varepsilon\right) & \text { if }-\varepsilon \leq t \leq 0, \\
-t & \text { if } t \leq-\varepsilon,\end{cases} \\
& \phi_{\varepsilon}^{3}(t)= \begin{cases}\varepsilon & \text { if } t \geq 0, \\
\sqrt{t^{2}+\varepsilon^{2}} & \text { if } t \leq 0 .\end{cases}
\end{aligned}
$$

With these choices, problem (2.7) admits a unique solution. To establish the convergence of the sequence $\left(u_{\varepsilon}\right)$, we need the following results (see [2]).

LEMmA 2.2. Let $V$ be a Hilbert space, $a: V \times V \rightarrow \mathbb{R}$ a continuous, $V$-elliptic bilinear, $j: V \rightarrow \mathbb{R}$ proper, nonnegative, convex, weakly continuous function, and $f$ a linear continuous on $V$. Assume that $j_{\varepsilon}: V \rightarrow \mathbb{R}, \varepsilon>0$, is a family of nonnegative convex weakly lower semicontinuous (l.s.c.) functions verifying

$$
\begin{gathered}
j_{\varepsilon}(v) \longrightarrow j(v) \quad \forall v \in V \\
\text { if } u_{\varepsilon} \longrightarrow u \text { weakly in } V, \text { then } j(u) \leq \liminf _{\varepsilon \rightarrow 0} j_{\varepsilon}\left(u_{\varepsilon}\right) .
\end{gathered}
$$

Let $u$ and $u_{\varepsilon}$ be the solutions of the following variational inequalities:

$$
\begin{gathered}
a(u, v-u)+j(v)-j(u)+\langle f, v-u\rangle \geq 0 \quad \forall v \in V, \\
a\left(u_{\varepsilon}, v-u_{\varepsilon}\right)+j_{\varepsilon}(v)-j_{\varepsilon}\left(u_{\varepsilon}\right)+\left\langle f, v-u_{\varepsilon}\right\rangle \geq 0 \quad \forall v \in V,
\end{gathered}
$$

respectively. Then, $u_{\varepsilon} \rightarrow u$ in $V$ when $\varepsilon \rightarrow 0$.

LEMMA 2.3. Assume that

$$
j(v)=\int_{\Omega} \phi(v) d x, \quad j_{\varepsilon}(v)=\int_{\Omega} \phi_{\varepsilon}(v) d x
$$

and $j$ is weakly l.s.c. If

$$
\phi_{\varepsilon}(t) \longrightarrow \phi(t) \text { uniformly in } t \text {, as } \varepsilon \longrightarrow 0 \text {, }
$$

then (2.11) is verified. 
We notice that if

$$
\left|\phi_{\varepsilon}(t)-\phi(t)\right| \leq c \varepsilon \quad \forall t \in \mathbb{R}
$$

then (2.14) is verified. Since the functions $\phi_{\varepsilon}^{j}, j=1,2,3$, verify inequality (2.15), then we have the convergence $u_{\varepsilon} \rightarrow u$ when $\varepsilon \rightarrow 0$ in $H^{1}(\Omega)$.

Taking $v=u_{\varepsilon}$ (resp., $v=u$ ) in the inequality of problem (2.1) (resp., (2.7)), we obtain

$$
a\left(u-u_{\varepsilon}, u-u_{\varepsilon}\right) \leq \varphi\left(u_{\varepsilon}\right)-\varphi_{\varepsilon}\left(u_{\varepsilon}\right)+\varphi_{\varepsilon}(u)-\varphi(u) .
$$

Consequently, we obtain the following a priori error estimates:

$$
\left\|u-u_{\varepsilon}\right\|_{H^{1}(\Omega)} \leq(2 c)^{1 / 2} \sqrt{\varepsilon} .
$$

3. A posteriori error estimates. In this section, we use the duality method by conjugating functions in order to derive the a posteriori error estimates of the solution of approximate problem. We need the preliminary results (see [1]).

Let $V$ and $V^{*}$ (resp., $Y$ and $Y^{*}$ ) be two topological vector spaces and $\langle\cdot, \cdot\rangle_{V}$ (resp., $\langle\cdot, \cdot\rangle_{Y}$ ) denote the duality pairing between $V$ and $V^{*}$ (resp., $Y$ and $Y^{*}$ ). Let $\varphi$ be a function from $V$ to $\overline{\mathbb{R}}=\mathbb{R} \cup\{-\infty,+\infty\}$, and let its conjugate function be defined by

$$
\varphi^{*}\left(v^{*}\right)=\sup _{v \in V}\left\langle v^{*}, v\right\rangle_{V}-\varphi(v)
$$

where $v^{*}$ is in $V^{*}$.

Assume that there exists a continuous linear operator $L$ from $V$ to $Y, L \in$ $\mathscr{L}(V, Y)$, with transpose $L^{*} \in \mathscr{L}\left(Y^{*}, V^{*}\right)$. Let $J$ be a function from $V \times Y$ to $\overline{\mathbb{R}}$.

We consider the following minimization problem:

$$
u \in V, \quad J(u, L u)=\inf _{v \in V} J(v, L v)
$$

where the conjugate function of $J$ is given by

$$
J^{*}\left(y^{*}, v^{*}\right)=\sup _{v \in V, y \in Y}\left\{\left\langle v^{*}, v\right\rangle_{V}+\left\langle y^{*}, y\right\rangle_{Y}-J(v, y)\right\}
$$

THeOREM 3.1. Assume that $V$ is a reflexive Banach space and $Y$ a normed vector space. Let $J: V \times Y \rightarrow \overline{\mathbb{R}}$ be a proper l.s.c. strictly convex function verifying 
(i) there exists $u_{0} \in V$ such that $J\left(u_{0}, L u_{0}\right)<\infty$ and $y \rightarrow J\left(u_{0}, y\right)$ is continuous at $L u_{0}$;

(ii) $J(v, L v) \rightarrow+\infty$ as $\|v\|_{V} \rightarrow+\infty, v \in V$.

Then, problem (3.2) admits a unique solution, and

$$
J(u, L u)=\inf _{v \in V} J(v, L v)=-\sup _{y^{*} \in Y^{*}} J^{*}\left(-y^{*}, L^{*} y^{*}\right)
$$

Let $\Omega$ be an open subset of $\mathbb{R}^{n}$, and $g: \Omega \times \mathbb{R}^{n} \rightarrow \mathbb{R}$ be the Carathéodory function, that is, for all $s \in \mathbb{R}^{n}, x \rightarrow g(x, s)$ is a measurable function, and, for almost all $x \in \Omega$, the function $s \rightarrow g(x, s)$ is continuous. Then, the conjugate function of

$$
G(v)=\int_{\Omega} g(x, v(x)) d x
$$

(assuming $G$ is well defined over some function space $V$ ) is

$$
G^{*}\left(v^{*}\right)=\int_{\Omega} g\left(x, v^{*}(x)\right) d x \quad \forall v^{*} \in V^{*},
$$

where

$$
g^{*}(x, y)=\sup _{s \in \mathbb{R}^{N}}\{y s-g(x, s)\}
$$

For problem (2.1), we take

$$
\begin{aligned}
V & =H^{1}(\Omega), \quad Y=Y^{*}=\left(L^{2}(\Omega)\right)^{n} \times L^{2}(\Omega), \\
L v & =(\nabla v, v), \quad J(v, L v)=H(v)+G(L v), \\
H(v) & = \begin{cases}0 & \text { if } v \geq 0 \text { on } \partial \Omega, \\
+\infty & \text { otherwise, }\end{cases} \\
G(y) & =\int_{\Omega} \frac{1}{2}\left|y_{1}\right|^{2}+\frac{1}{2}\left|y_{2}\right|^{2}+f y_{2},
\end{aligned}
$$

where $y=\left(y_{1}, y_{2}\right)$ with $y_{1} \in\left(L^{2}(\Omega)\right)^{n}$ and $y_{2} \in L^{2}(\Omega)$; a similar notation is used for $y^{*} \in Y^{*}$. So, the obstacle problem (2.1) can be rewritten in the form (3.2).

To apply Theorem 3.1 , we compute the conjugate of the functional $J$. We have

$$
J^{*}\left(-y^{*}, L^{*} y^{*}\right)=H^{*}\left(L^{*} y^{*}\right)+G^{*}\left(-y^{*}\right),
$$


where

$$
\begin{aligned}
H^{*}\left(L^{*} y^{*}\right) & =\sup _{v \in H^{1}(\Omega)}\{\langle L v, y\rangle-H(v)\} \\
& =\sup _{\left\{v \in H^{1}(\Omega), v \geq 0 \text { in } \Gamma\right\}} \int_{\Omega}\left(\nabla v y_{1}^{*}+v y_{2}^{*}\right) d x \\
& = \begin{cases}0 & \text { if }-\operatorname{div} y_{1}^{*}+y_{2}^{*}=0 \text { in } \Omega, \\
\infty & \text { otherwise, }\end{cases} \\
G^{*}\left(-y^{*}\right) & =\sup _{y \in Y}\left\{\left\langle-y^{*}, y\right\rangle-G(y)\right\} \\
& =\sup _{y \in Y} \int_{\Omega}\left(-y_{1}^{*} y_{1}-y_{2}^{*} y_{2}-\frac{1}{2}\left|y_{1}\right|^{2}-\frac{1}{2}\left|y_{2}\right|^{2}-f y_{2}\right) d x \\
& =\int_{\Omega}\left(\frac{1}{2}\left|y_{1}^{*}\right|^{2}+\frac{1}{2}\left|y_{2}^{*}-f\right|^{2}\right) d x .
\end{aligned}
$$

Hence,

$$
J^{*}\left(-y^{*}, L^{*} y^{*}\right)= \begin{cases}\int_{\Omega}\left(\frac{1}{2}\left|y_{1}^{*}\right|^{2}+\frac{1}{2}\left|y_{2}^{*}-f\right|^{2}\right) d x & \text { if }-\operatorname{div} y_{1}^{*}+y_{2}^{*}=0 \\ \infty & \text { otherwise. }\end{cases}
$$

We have

$$
\begin{aligned}
& J\left(u_{\varepsilon}, L u_{\varepsilon}\right)-J(u, L u)=\int_{\Omega} \frac{1}{2}\left|\nabla u_{\varepsilon}\right|^{2}-\frac{1}{2}|\nabla u|^{2}+\frac{1}{2}\left|u_{\varepsilon}\right|^{2} \\
& -\frac{1}{2}|u|^{2}+f\left(u_{\varepsilon}-u\right) d x \text {. }
\end{aligned}
$$

Using (2.8) with $v=u_{\varepsilon}$, we obtain

$$
J\left(u_{\varepsilon}, L u_{\varepsilon}\right)-J(u, L u) \geq \frac{1}{2}\left\|u_{\varepsilon}-u\right\|_{H^{1}(\Omega)}^{2} .
$$

Applying Theorem 3.1 and using (3.11), we have

$$
\begin{aligned}
J\left(u_{\varepsilon}, L u_{\varepsilon}\right)-J(u, L u) \leq \int_{\Omega} & \frac{1}{2}\left|\nabla u_{\varepsilon}\right|^{2}+\frac{1}{2}\left|u_{\varepsilon}\right|^{2}+f u_{\varepsilon} \\
& +\frac{1}{2}\left|y_{1}^{*}\right|^{2}+\frac{1}{2}\left|y_{2}^{*}-f\right|^{2} d x
\end{aligned}
$$

for all $y^{*}=\left(y_{1}^{*}, y_{2}^{*}\right) \in Y^{*}$, with $-\operatorname{div} y_{1}^{*}+y_{2}^{*}=0$ in $\Omega$.

Since $\phi_{\varepsilon}$ is differentiable, inequality (2.9) is equivalent to $u_{\varepsilon} \in H^{1}(\Omega)$

$$
a\left(u_{\varepsilon}, v\right)+\int_{\Gamma} \phi_{\varepsilon}^{\prime}\left(u_{\varepsilon}\right) v+\int_{\Omega} f v=0
$$


Hence, $u_{\varepsilon}$ verifies the following Neumann problem:

$$
\begin{array}{ll}
-\Delta u_{\varepsilon}+u_{\varepsilon}+f=0 & \text { in } \Omega, \\
\frac{\partial u_{\varepsilon}}{\partial n}=-\int_{\Gamma} \phi_{\varepsilon}^{\prime}\left(u_{\varepsilon}\right) & \text { on } \Gamma .
\end{array}
$$

If we take

$$
y_{1}^{*}=\nabla u_{\varepsilon}, \quad y_{2}^{*}=\left(u_{\varepsilon}+f\right) u_{\varepsilon},
$$

then we have

$$
-\operatorname{div} y_{1}^{*}+y_{2}^{*}=0
$$

Then, we have the a posteriori error estimate

$$
\frac{1}{2}\left\|u_{\varepsilon}-u\right\|_{H^{1}(\Omega)}^{2} \leq \int_{\Omega}\left|\nabla u_{\varepsilon}\right|^{2}+\left|u_{\varepsilon}\right|^{2}+f u_{\varepsilon} .
$$

Taking $v=u_{\varepsilon} \in H^{1}(\Omega)$ in (3.15),

$$
a\left(u_{\varepsilon}, u_{\varepsilon}\right)+\int_{\Gamma} \phi_{\varepsilon}^{\prime}\left(u_{\varepsilon}\right)\left(u_{\varepsilon}\right)+\int_{\Omega} f u_{\varepsilon}=0 .
$$

Hence,

$$
\int_{\Omega}\left|\nabla u_{\varepsilon}\right|^{2}+\int_{\Omega}\left|u_{\varepsilon}\right|^{2}+\int_{\Gamma} \phi_{\varepsilon}^{\prime}\left(u_{\varepsilon}\right)\left(u_{\varepsilon}\right)+\int_{\Omega} f u_{\varepsilon}=0 .
$$

Estimate (3.19) becomes

$$
\frac{1}{2}\left\|u_{\varepsilon}-u\right\|_{H^{1}(\Omega)}^{2} \leq \int_{\Gamma} \phi_{\varepsilon}^{\prime}\left(u_{\varepsilon}\right)\left(u_{\varepsilon}\right)
$$

Hence, we obtain the a posteriori error estimates.

For the choice (1.1) and (1.2), we have

$$
\phi_{\varepsilon}^{\prime}(t)= \begin{cases}0 & \text { if } t \geq 0 \\ \frac{t}{\varepsilon} & \text { if }-\varepsilon \leq t \leq 0 \\ -1 & \text { if } t \leq-\varepsilon\end{cases}
$$

The a posteriori error estimate is

$$
\frac{1}{2}\left\|u_{\varepsilon}-u\right\|_{H^{1}(\Omega)}^{2} \leq \int_{\left[u_{\varepsilon} \leq-\varepsilon\right]}\left|u_{\varepsilon}\right|+\int_{\left[-\varepsilon \leq u_{\varepsilon} \leq 0\right]} \frac{\left|u_{\varepsilon}\right|^{2}}{\varepsilon} .
$$


For the choice (2.4), we have

$$
\phi_{\varepsilon}^{\prime}(t)= \begin{cases}0 & \text { if } t \geq 0, \\ \frac{t}{\sqrt{t^{2}+\varepsilon^{2}}} & \text { if } t \leq 0 .\end{cases}
$$

The a posteriori error estimate is

$$
\frac{1}{2}\left\|u_{\varepsilon}-u\right\|_{H^{1}(\Omega)}^{2} \leq \int_{\left[u_{\varepsilon} \leq 0\right]} \frac{\left|u_{\varepsilon}\right|^{2}}{\sqrt{u_{\varepsilon}^{2}+\varepsilon^{2}}}
$$

4. Application. Consider the following variational inequality problem:

$$
\text { Find } u \in K, \quad a(u, v-u)+\langle f, v-u\rangle \geq 0 \quad \forall v \in K \text {, }
$$

where $a(\cdot, \cdot)$ is defined by

$$
\begin{aligned}
a(u, v) & =\int_{\Omega} \nabla u \cdot \nabla v+\int_{\Omega} u \cdot v, \quad u, v \in H^{1}(\Omega), \\
K & =\left\{v \in H^{1}(\Omega): v \geq 0 \text { on } \Gamma\right\} .
\end{aligned}
$$

It is well known that problem (4.1) admits a unique solution (see [3]).

We write the obstacle problem (4.1) in a new form.

THEOREM 4.1. Let $\left(u_{\alpha}\right)(\alpha \geq 0, \alpha \rightarrow 0)$ be the solution of the following problem-called penalised problem:

$$
\begin{aligned}
& \text { Find } u_{\alpha} \in H^{1}(\Omega), \\
& a\left(u_{\alpha}, v-u_{\alpha}\right)+\varphi_{\alpha}(v)-\varphi_{\alpha}\left(u_{\alpha}\right)+\left\langle f, v-u_{\alpha}\right\rangle \geq 0 \quad \forall v \in H^{1}(\Omega),
\end{aligned}
$$

where $\varphi_{\alpha}$ is the functional defined by

$$
\varphi_{\alpha}(v)=\frac{1}{\alpha} \int_{\Gamma} v^{-}, \quad v \in H^{1}(\Omega)
$$

Problem (4.3) admits a unique solution and $\left(u_{\alpha}\right)_{(\alpha \geq 0)}$ solution of (4.3) converges to $u$ solution of (4.1) when $\alpha \rightarrow 0$.

Proof (see [2]). The functional $\varphi_{\alpha}$, being nondifferentiable on $H^{1}(\Omega)$, is approximated by a sequence of differentiable functionals

$$
\varphi_{\alpha, \varepsilon}(v)=\frac{1}{\alpha} \int_{\Gamma} \phi_{\varepsilon}(v) d x, \quad(\varepsilon \geq 0, \varepsilon \longrightarrow 0) .
$$


The regularized problem is

$$
\begin{aligned}
& \text { Find } u_{\alpha, \varepsilon} \in H^{1}(\Omega), \\
& a\left(u_{\alpha, \varepsilon}, v-u_{\alpha, \varepsilon}\right)+\varphi_{\alpha, \varepsilon}(v)-\varphi_{\alpha, \varepsilon}\left(u_{\alpha, \varepsilon}\right)+\left\langle f, v-u_{\alpha, \varepsilon}\right\rangle \geq 0 \quad \forall v \in H^{1}(\Omega) .
\end{aligned}
$$

Problems (4.3) and (4.6) are, respectively, equivalent to

$$
\begin{aligned}
u_{\alpha} \in H^{1}(\Omega): & a\left(u_{\alpha}, v-u_{\alpha}\right)+\frac{1}{\alpha} \int_{\Gamma}\left(v^{-}-u_{\alpha}^{-}\right) d x \\
+ & \int_{\Omega} f\left(v-u_{\alpha}\right) d x \geq 0 \quad \forall v \in H^{1}(\Omega), \\
u_{\alpha, \varepsilon} \in H^{1}(\Omega): & a\left(u_{\alpha, \varepsilon}, v-u_{\alpha, \varepsilon}\right)+\varphi_{\alpha, \varepsilon}(v)-\varphi_{\alpha, \varepsilon}\left(u_{\alpha, \varepsilon}\right) \\
+ & \int_{\Omega} f\left(v-u_{\alpha, \varepsilon}\right) d x \geq 0 \quad \forall v \in H^{1}(\Omega) .
\end{aligned}
$$

We can similarly proceed to drive the a posteriori error estimates; then, for the choice (1.1) and (1.2), we have

$$
\phi_{\varepsilon}^{\prime}(t)= \begin{cases}0 & \text { if } t \geq 0 \\ \frac{t}{\varepsilon} & \text { if }-\varepsilon \leq t \leq 0 \\ -1 & \text { if } t \leq-\varepsilon\end{cases}
$$

The a posteriori error estimate is

$$
\frac{1}{2}\left\|u_{\alpha, \varepsilon}-u_{\alpha}\right\|_{H^{1}(\Omega)}^{2} \leq \frac{1}{\alpha} \int_{\left[u_{\alpha, \varepsilon} \leq-\varepsilon\right]}\left|u_{\alpha, \varepsilon}\right|+\frac{1}{\alpha} \int_{\left[-\varepsilon \leq u_{\alpha, \varepsilon} \leq 0\right]} \frac{\left|u_{\alpha, \varepsilon}\right|^{2}}{\varepsilon} .
$$

For the choice (2.4), the a posteriori error estimate is

$$
\frac{1}{2}\left\|u_{\alpha, \varepsilon}-u_{\alpha}\right\|_{H^{1}(\Omega)}^{2} \leq \frac{1}{\alpha} \int_{\left[u_{\alpha, \varepsilon} \leq 0\right]} \frac{\left|u_{\alpha, \varepsilon}\right|^{2}}{\sqrt{u_{\alpha, \varepsilon}^{2}+\varepsilon^{2}}}
$$

5. A posteriori error estimates for regularized discrete problem. Let $V_{h}$ be a finite element space approximating $H^{1}(\Omega)$. Then, the finite element solution $u_{h} \in V_{h}$ for the obstacle problem (4.1) is determined from the following problem:

$$
\begin{aligned}
& \text { Find } u_{h} \in V_{h}, u_{h} \geq 0 \text { on } \partial \Omega \\
& a\left(u_{h}, v_{h}-u_{h}\right)+\left\langle f, v_{h}-u_{h}\right\rangle \geq 0 \quad \forall v_{h} \in V_{h}, v_{h} \geq 0 \text { on } \partial \Omega .
\end{aligned}
$$


If we use the penalisation method, then the solution $u_{h}$ of problem (5.1) is the limit when $\alpha$ tends to 0 of the solution $u_{\alpha, h}$ of the following problem:

$$
\begin{aligned}
& \text { Find } u_{\alpha, h} \in V_{h}, \\
& a\left(u_{\alpha, h}, v_{h}-u_{\alpha, h}\right)+\varphi_{\alpha}\left(v_{h}\right)-\varphi_{\alpha}\left(u_{\alpha, h}\right)+\left\langle f_{h}, v_{h}-u_{\alpha, h}\right\rangle \geq 0 \quad \forall v_{h} \in V_{h} .
\end{aligned}
$$

We can similarly proceed as in [2] to prove the convergence of the finite element approximations and to have a priori error estimates.

The regularized problem of (5.2) is

$$
\begin{aligned}
& \text { Find } u_{\alpha, h, \varepsilon} \in V_{h}, \\
& a\left(u_{\alpha, h, \varepsilon}, v_{h}-u_{\alpha, h, \varepsilon}\right)+\varphi_{\alpha, \varepsilon}\left(v_{h}\right)-\varphi_{\alpha, \varepsilon}\left(u_{\alpha, h, \varepsilon}\right)+\left\langle f_{h}, v_{h}-u_{\alpha, h, \varepsilon}\right\rangle \geq 0 \quad \forall v_{h} \in V_{h} .
\end{aligned}
$$

We can similarly prove that (5.3) has unique solution and its solution converges to the corresponding solution of problem (5.2).

By the duality theory on the discrete problems, we prove the following a posteriori error estimates.

For the choice (1.1) and (1.2), the a posteriori error estimate is

$$
\frac{1}{2}|| u_{\alpha, h, \varepsilon}-\left.u_{\alpha, h}\right|_{H^{1}(\Omega)} ^{2} \leq \frac{1}{\alpha} \int_{\left[u_{\alpha, h, \varepsilon} \leq-\varepsilon\right]}\left|u_{\alpha, h, \varepsilon}\right|+\frac{1}{\alpha} \int_{\left[-\varepsilon \leq u_{\alpha, h, \varepsilon} \leq 0\right]} \frac{\left|u_{\alpha, h, \varepsilon}\right|^{2}}{\varepsilon} .
$$

For the choice (2.4), the a posteriori error estimate is

$$
\frac{1}{2}\left\|u_{\alpha, h, \varepsilon}-u_{\alpha, h}\right\|_{H^{1}(\Omega)}^{2} \leq \frac{1}{\alpha} \int_{\left[u_{\alpha, h, \varepsilon} \leq 0\right]} \frac{\left|u_{\alpha, h, \varepsilon}\right|^{2}}{\sqrt{u_{\alpha, h, \varepsilon}^{2}+\varepsilon^{2}}}
$$

\section{REFERENCES}

[1] I. Ekeland and R. Temam, Analyse Convexe et Problèmes Variationnels, Collection Études Mathématiques, Gauthier-Villars, Paris, 1974 (French).

[2] R. Glowinski, J.-L. Lions, and R. Trémolières, Numerical Analysis of Variational Inequalities, Studies in Mathematics and Its Applications, vol. 8, NorthHolland Publishing, Amsterdam, 1981.

[3] D. Kinderlehrer and G. Stampacchia, An Introduction to Variational Inequalities and Their Applications, Pure and Applied Mathematics, vol. 88, Academic Press, New York, 1980.

A. Addou: Department of Mathematics, Faculty of Sciences, Mohammed I University, Oujda, Morocco

J. Zahi: Department of Mathematics, Faculty of Sciences, Mohammed I University, Oujda, Morocco

E-mail address: zahi-j@sciences.univ-oujda.ac.ma 


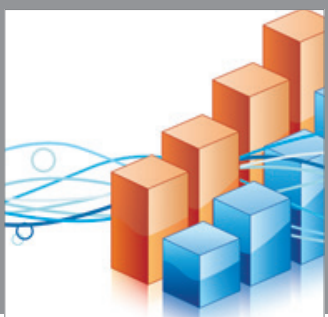

Advances in

Operations Research

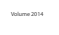

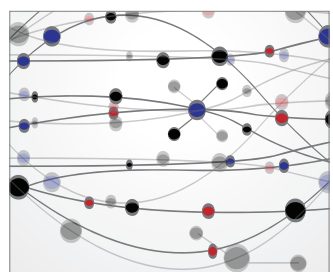

\section{The Scientific} World Journal
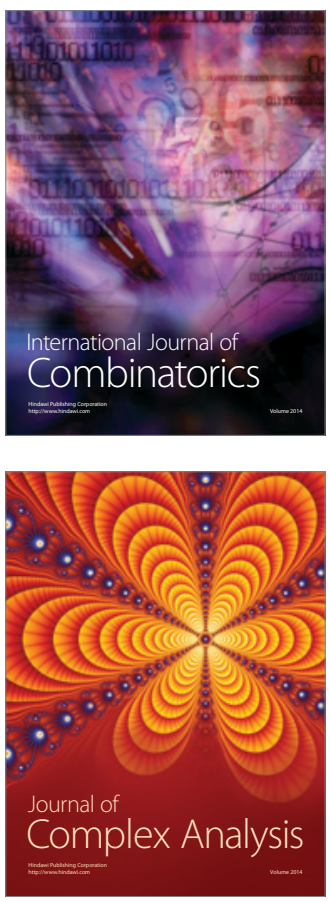

International Journal of

Mathematics and

Mathematical

Sciences
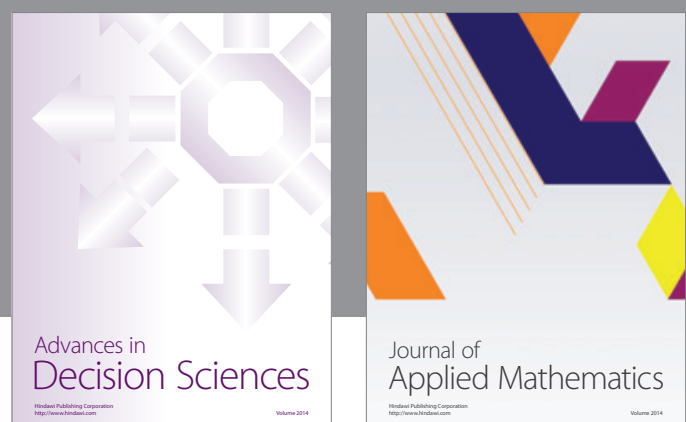

Journal of

Applied Mathematics
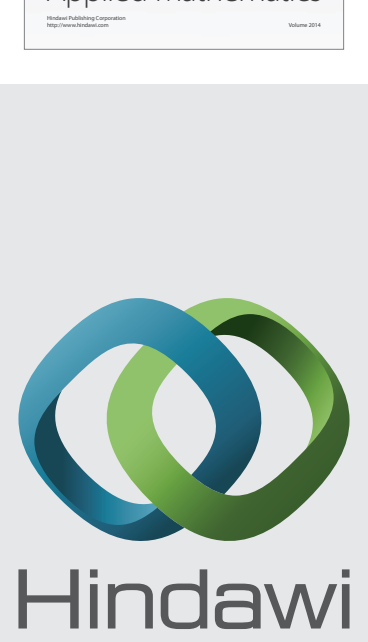

Submit your manuscripts at http://www.hindawi.com
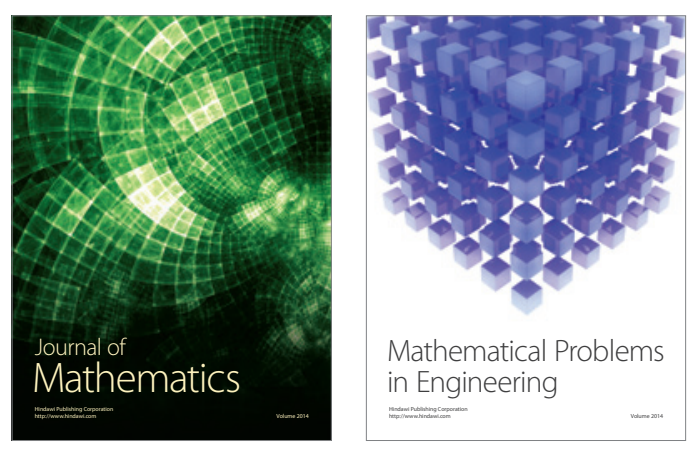

Mathematical Problems in Engineering
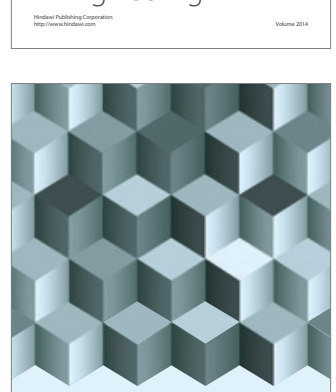

Journal of

Function Spaces
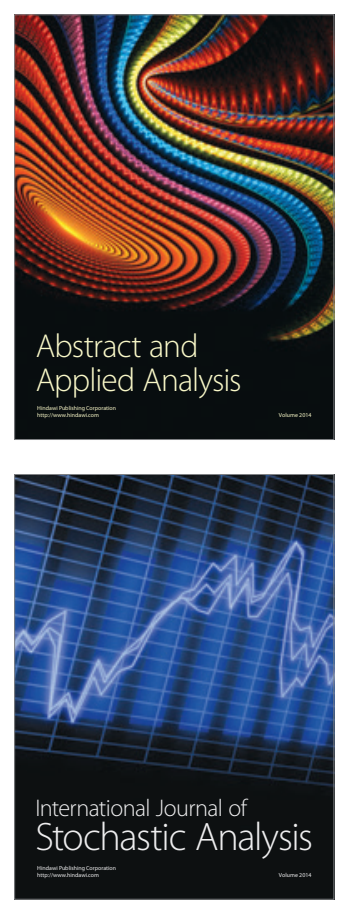

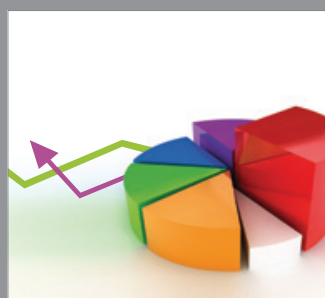

ournal of

Probability and Statistics

Promensencen
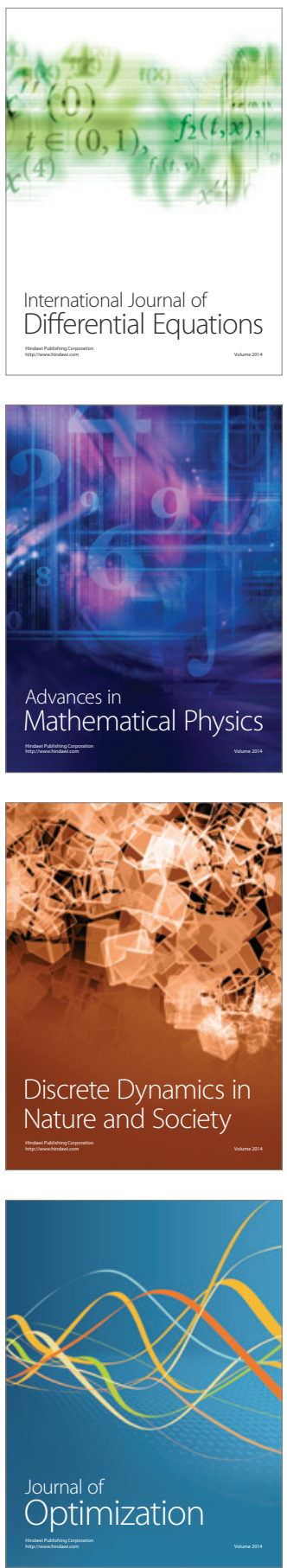